\title{
Optimal Management of the Elderly Patient with Muscle-Invasive Bladder Cancer
}

\author{
Eugene J. Pietzak • George W. Drach • \\ S. Bruce Malkowicz • Thomas J. Guzzo
}

Published online: 20 March 2013

(C) Springer Science+Business Media New York 2013

\begin{abstract}
With the growing elderly demographic and the peak incidence of bladder cancer being approximately 70 years old, an increasing number of elderly patients will develop invasive bladder cancer. Several studies suggest that a survival gap exists between elderly patients with invasive bladder cancer and younger cohorts. Radical cystectomy is the standard of care that provides good cancer-specific survival, but few elderly patients are referred for cystectomy. This is likely, at least in part, due to concern with the morbidity associated with the operation. Cisplatin is the most effective chemotherapeutic agent used to treat invasive bladder cancer but some patients cannot receive it due to toxicity. There are few promising alternatives to cisplatin at this time. This review discusses opportunities for improved care, recent therapeutic advances, and future directions to improve the outcome for elderly patients with invasive bladder cancer.
\end{abstract}

Keywords Muscle invasive bladder cancer - Elderly · Radical cystectomy $\cdot$ Cisplatin · Cisplatin unfit · Urinary diversion · Bladder sparing $\cdot$ Bladder cancer $\cdot$ Urothelial carcinoma $\cdot$ Radical TURBT

\section{Introduction}

There were approximately 73,510 new cases of bladder cancer in the United States in 2012 [1]. With the incidence of bladder cancer increasing with age, it is most commonly seen in patients older than 70 years of age [1]. While bladder

\footnotetext{
E. J. Pietzak $(\bowtie) \cdot$ G. W. Drach • S. B. Malkowicz • T. J. Guzzo Division of Urology, Hospital of the University of Pennsylvania, 3400 Civic Blvd, 3rd floor West Perelman Center for Advanced Medicine, Philadelphia, PA 19104, USA

e-mail: eugene.pietzak@uphs.upenn.edu
}

cancer is the sixth most common cancer overall, it is the fourth most common in patients older than 85 years [2]. Fortunately, the vast majority of tumors will not invade into the muscle of the bladder wall. These non-invasive tumors can often be managed with conservative techniques, such as transurethral resection of the bladder tumor (TURBT) and intravesical therapies.

However, some of patients with non-muscle invasive tumors will progress to muscle invasive disease and approximately $20 \%$ of patients will have invasive disease at initial presentation. Even with the best available treatments, the overall survival for muscle invasive bladder cancer (MIBC) is remains only $40-60 \%$ at 5 years [3]. An estimated 14,880 patients died from bladder cancer in 2012 in the United States [1].

The peak age for MIBC is 70 years of age with $20 \%$ of patients being over 80 years old [4]. As the United States' elderly population continues to grow at an unprecedented rate an increasing number of elderly will develop MIBC [2]. Similarly, elderly patients with non-muscle invasive tumors are less likely to respond to intravesical therapy, which puts them at greater risk of progression to MIBC [5].

Yet, several studies have shown a disparity in both cancer care and outcomes between elderly patients with MIBC compared to younger patients. There are multiple reasons for why these differences may exist. It is the objective of this review article to highlight areas that need most improvement in the care of elderly patients with MIBC and to discuss recent advances as well as future directions.

\section{Radical Cystectomy in the Elderly: An Under-Utilized Standard of Care}

Radical cystectomy with urinary diversion (RC) offers the best chance of survival for patients with MIBC and remains 
the gold standard for which alternative therapies are compared. The 10-year cancer-specific survival and overall survival for RC is approximately $70 \%$ and $50 \%$, respectively, for organ-confined disease [6]. Patients over 75 and 80 years have been successfully treated with RC with similar cancer specific survival to younger cohorts [7•, 8-11]. However, some studies have reported that older patients have more aggressive tumors than younger patients $[12,13]$. However, when reviewing the care that elderly patients with MIBC receive, obvious disparities appear. For example, elderly patients with MIBC are less likely to receive aggressive treatments, even though aggressive treatment has been shown to improve survival in patients older than 80 years $[13,14 \cdot 15]$. Similarly, elderly patients are less likely to get an extended lymph node dissection, which is also associated with improved outcomes [16, 17]. Lymphadenectomy in the elderly may be avoided by some surgeons due the increased time under anesthesia and concern about dissecting near atherosclerotic vessels. Although not yet proven in randomized controlled trials, retrospective studies suggest extended lymphadenectomy may have a therapeutic benefit and the number of lymph nodes removed may be an indictor of surgical quality $[4,18]$. Surgical series have reported that when a similar number of nodes are removed from the elderly, there is comparable cancer specific survival to younger cohorts $[17,18]$.

One important area that warrants further investigation is that although cancer specific outcomes appear similar between older and younger cohorts, the overall survival is usually significant worse for elderly patients. This may result from competing risks due to differences in comorbidity and age-related changes. However it could be related to the impact that $\mathrm{RC}$ has on the elderly. $\mathrm{RC}$ is a major surgery with multiple suture lines, an intestinal anastomosis, abdominal incisions, and urinary diversion required for this operation. RC may result in a physiologic insult that could worsen or expose frailty within an individual [19].

Despite recent advances in surgical technique and peri-operative management that have substantially reduced the mortality associated with the operation, the morbidity rate remains alarmingly high 19-64\% [20••, 21-23]. Those at greatest risk for morbidity and mortality after RC are those with multiple co-morbid conditions as well as the elderly [24, 25]. The risk of death within 90 days from $\mathrm{RC}$ is far greater in those $>80$ years old [26•]. However, multiple studies contest that it is not chronologic age, but physiologic age that matters most. It may simply be that older patients have had more time to compound comorbidities and if properly controlling for comorbidities, elderly patients will have similar outcomes to younger patients. Unfortunately, it is often difficult to adjust for comorbid conditions in studies.
For example, the commonly used Charlson Comorbidity score does not account for severity of comorbid conditions and the American Society of Anesthesia (ASA) score may not be sensitive enough to properly discriminate differences $[27,28]$.

Elderly patients being considered for RC should undergo an individualized assessment of life expectancy, comorbidities, and functional status [29]. Various competing risks for the patient should also be considered. Chronologic age should be factored in to a lesser extent, as age often does not accurate predict ability to tolerate treatments [29]. Using an assessment tool designed specifically for elderly cancer patients may be most appropriate, albeit, also time consuming. Use of the preoperative assessment in elderly cancer patients (PACE) or the Comprehensive Geriatric Assessment should be considered before surgery as properly selected elderly patients are able to tolerate RC as well as younger patients [30, 31].

Considering that $\mathrm{RC}$ is the standard of care for MIBC, it is considerably under-utilized [16, 32]. Studies show that approximately half of patients between the ages 55-59 years with MIBC undergo RC, and those numbers rapidly decrease with increasing age [33]. For patients ages 85 years and older, only $4 \%$ will undergo a RC. When looking at only those with few comorbidities (ASA score $\leq 2$ ), only $9 \%$ will receive a RC [33]. Under-utilization is not necessarily because surgeons are declining to perform the operation in this older cohort, as less than $5 \%$ of patients referred for cystectomy are considered unfit by experienced surgeons [34].

The low referral rate of MIBC patients may reflect a bias in primary care providers against aggressive treatment in elderly patients. Primary care providers, including primary urologists, often influence the decision for an elderly patient to undergo RC, but often underestimate the patient's willingness to undergo aggressive treatment $[35,36]$. While this is well intentioned, it can result in a significant delay in definitive treatment. Delays of over 12 weeks from diagnosis to RC have been shown to be detrimental to outcomes [37]. This delay may partially explain the worse pathological findings seen in some elderly patients undergoing RC $[12,13]$.

Future efforts are needed to focus on facilitating referral of elderly patients with MIBC to high volume tertiary care centers. A volume-outcome relationship has been demonstrated for RC with high-volume centers providing superior outcomes $[20 \bullet \bullet, 38]$. Academic centers are also able to provide multi-disciplinary care, which is of increasing importance in this disease. A noted barrier for a patient undergoing $\mathrm{RC}$ is long distances from a cancer center [39]. This is an important consider in the elderly, as they may depend on others for transportation to physician appointments. 


\section{Optimizing Radical Cystectomy Outcomes in Elderly Patients}

As previously mentioned, the morbidity associated with RC is high and potentially worse for elderly patients. A prolonged postoperative ileus (PPOI) is the most common complication following RC [22]. Although, there is no standardized definition for PPOI and the pathophysiology contributing to this process is rather complex, several attempts have been made at decreasing the incidence of PPOI, ranging from pharmacologic agents to gum chewing [40, 41]. PPOI has previously been shown to be a common cause of a prolonged length of hospital stay (LOS) following RC. When we evaluated our RC experience of 330 consecutive patients for perioperative risk factors associated with a prolonged LOS $\geq 12$ days, only older age and female gender were statistical significant on multivariate analysis [42]. Those with a prolonged LOS had significantly similar cancer specific outcomes but worse overall survival [42].

Another important consideration in the elderly is the rate of delirium following RC. It has recently been shown to be $29 \%$ following RC in patients older than 65 years [43]. Delirium is associated with an increased risk of morbidity and mortality after many surgical procedures [44]. Unfortunately, it is often under detected by health care providers. Evidence suggests that the involvement of geriatricians with in-patient care after surgery may decrease the risk of postoperative delirium [45].

With recent changes in the United States health care system, there has been a well-documented shift toward earlier discharges from the hospital after RC by discharging to skilled care facilities [23, 46]. However, patients discharged to a skilled care facility are at much greater risk of morbidity and mortality after RC as well as many other surgeries [47]. It should not come as a surprise that elderly patients are more likely to be discharged to a skilled care facility after $\mathrm{RC}$ and further research in this area is needed to more clearly define the role of such facilities following RC in elderly populations [48].

The readmission rate after RC is approximately $20-30 \%$ within the first 90 days. In fact, RC has higher readmission rates than esophagectomy and pancreatectomy [49]. Those with comorbidities are at increased risk for readmission after RC [22]. Dehydration and failure to thrive are a common cause of readmission [22]. With greater attention being paid to the concept of frailty and geriatric failure to thrive, preemptive action may decrease hospital admission rates in this population [19]. Family involvement in the care of elderly patients after RC will also play a role in decreasing the risk of readmission. When planning to discharge an elderly patient from the hospital after RC, level of social support should be considered. For example, elderly widowed men are at an increased risk for worse outcomes after RC [50].

It is important to remember that it could take several months for an elderly patient to recover from major abdominal surgery, both cognitively and physically [51]. RC is no different in this regard, with loses of lean body mass and strength seen up to 6 months later [52]. A target area for improving outcomes following $\mathrm{RC}$ is nutritional support. Approximately $20 \%$ of patients undergoing RC have evidence of malnourishment [53, 54]. Although, not entirely specific for malnourishment, low serum albumin levels is one of strongest predictors of morbidity and mortality [55]. This is also true in the RC literature [26•]. Seven to ten days of preoperative oral nutritional supplementation has shown to benefit gastrointestinal cancer patients undergoing surgery [56]. Peri-operative nutritional support will likely have a future role in optimizing outcomes for RC, particularly in the elderly and malnourished.

Additional future directions for improving RC outcomes will focus on reducing the morbidity of the procedure. The major contributor to morbidity is the use of bowel in the urinary diversion. One consideration is the use of cutaneous ureterostomies, which had fallen out of favor, but may have an increasing role with improvements in surgical techniques to help avoid bowel work [57]. Ultimately, the future of urinary diversion will likely be in tissue engineering [58-60]. The use of robotic assisted surgery is also gaining popularity, which may potentially decrease morbidity. Early reports suggest that robotic cystectomy is safe in elderly patients with similar short-term oncologic outcomes with a reduction in operative blood loss and a shorter LOS [61-63]. A combination of robotic assisted surgery and tissue engineered urinary diversions has the potential to markedly reduce the morbidity of RC [58].

\section{Urinary Diversions: Considerations for Elderly Patients}

Although continent diversions and orthotopic neobladders are increasingly being used at high volume centers, the most common diversion remains a non-continent diversion [64]. This is holds true in the elderly population [11, 64]. There has yet to be a well documented major difference in quality of life between the various urinary diversion types [65]. However, either a continent diversion or an orthotopic neobladder is a safe and reasonable option for carefully selected elderly patients [66].

There are several benefits to an ileal conduit diversion, which is likely the reason it is commonly used in the elderly population. The procedure has relative ease, shorter operative time, and more predictable functional outcomes [67]. The shorter length of contact time between urine and bowel results in less metabolic changes, although up to $10 \%$ with 
an ileal conduit will have some metabolic disturbances [67]. The most common metabolic disturbance is a hyperchloremic metabolic acidosis, which results from a chronic acid load and reduced renal function $[67,68]$. This can result in muscle weakness, electrolyte abnormalities, and bone demineralization. The resultant bone demineralization is of particular concern in the elderly. It occurs from the effects of chronic acidosis on bone buffing, an impairment of renal activation of vitamin $\mathrm{D}$, and an activation of osteoclasts [67]. To date there is no standardized way to follow or screen patients with urinary diversions for osteopenia [68, 69].

Another important consideration when selecting the type of urinary diversion in the elderly population is the individual's manual dexterity as well as their willingness and ability to properly maintain their diversion [70]. Early referral to a stoma care specialist is particularly important to optimize care and improve quality of life [68, 71]. Both family members and care providers should be encouraged to attend these appointments as many elderly patients will not independently care for their urostomy [72].

\section{Chemotherapy in Muscle Invasive Disease}

Cisplatin combination therapy remains the standard chemotherapeutic agent for urothelial cell carcinoma. Although initial trials used combination methotrexate, vinblastine, doxorubicin, and cisplatin (MVAC), more recently gemecitibine and cisplatin (GC) combination therapy has become the preferred regiment at many centers due to the similar efficacy to MVAC but with less toxicity [73•].

Neoadjuvant cisplatin combination therapy has a small but definite advantage in the treatment of MIBC with a $5 \%$ improved overall survival [74]. The rate of pT0 disease at time of cystectomy also increases from $10-15 \%$ with transurethral resection alone to $35-45 \%$ with neoadjuvant chemotherapy [73•]. Although pT0 does not guarantee cure, these patients do have a more favorable prognosis [75]. These potential benefits of neoadjuvant therapy must be weighed against the delay to $\mathrm{RC}$ that may result from non-responder or toxicity from therapy.

Prospective trials evaluating adjuvant chemotherapy for bladder cancer have suffered from methodological flaws and are limited by small subject numbers due to poor accrual [76-78]. Adjuvant chemotherapy is typically reserved for patients with $\geq \mathrm{pT} 3$ or positive nodes. This strategy may avoid overtreatment in pT0 disease patients and potentially pT2 patients. However, up to $17 \%$ patients following their urinary diversion will no longer for eligible for cisplatin due to impair renal function [79]. Only one prospective randomized trial attempted to compare neoadjuvant to adjuvant therapy, which showed no difference between them, but small subject numbers and study design limits interpretation [80].

Despite level one evidence demonstrating a benefit for neoadjuvant chemotherapy, only $1.2 \%$ of patients with MIBC will receive preoperative chemotherapy and only $11.6 \%$ of patients will receive any peri-operative chemotherapy [81]. Compounding this issue is that fact that approximately $40 \%$ of patients with MIBC are not "eligible" for cisplatin and no alternative systemic agent has similar efficacy. Recently, a consensus criteria was published on "fitness" to receive cisplatin; ECOG performance status of $\leq 2$, glomerular filtration rate $(\mathrm{GFR}) \geq 60 \mathrm{ml} / \mathrm{min}$, minimal comorbidities, particularly heart failure $[82 \bullet \bullet]$. The elderly not only have an increased rate of comorbid conditions, but also GFR decreases roughly $1 \%$ per year from about 30 years of age $[82 \bullet \cdot]$. Even if criteria for cisplatin are met by an elderly patient it is questionable as to whether the results of those clinical trials are generalizable to them as most trials limited enrollment to less than 70 years old. This is not a problem unique to bladder cancer as the elderly are frequently excluded from randomized control trials [83].

Finding an alternative therapy for cisplatin that is better tolerated and safe for "unfit" patients should be a top priority in bladder cancer. Carboplatin does have some activity against urothelial cell carcinoma but is far less effective and should not be considered a substitution for cisplatin. Carboplatin with gemcitabine may be the best option currently [84]. However, active investigation into alternatives is underway. Current trials are looking at various combination therapies, including the use of taxanes, novel microtubules inhibitors, folate synthesis inhibitors, and molecularly targeted agents $[84,85]$. Future efforts will also need to focus on translational research into tumor genetics to predict chemotherapy responders and help guide more targeted therapies [73•].

\section{Bladder Sparing Protocols}

Bladder sparing as an alternative to $\mathrm{RC}$ has gone through an extensive evolution [3]. All modern bladder sparing protocols use a trimodal therapy (TMT) approach of radical TURBT, chemotherapy, and radiation. Any of those modalities as a monotherapy alone produce inferior results. However, long-term results for TMT have shown a complete response rate in $60-80 \%$ of patients with a 5 -year overall survival between 40-60\% [3]. Although these results are comparable to RC series, it must be remembered that patients enrolled in most bladder sparing protocols are highly selected patients. Patients who are the most optimal candidate for TMT do not have diffuse carcinoma in situ (CIS), 
no hydroureteronephrosis, clinically negative lymph nodes, complete transurethral resection, minimal comorbidities, and enough renal function to tolerate cisplatin combination chemotherapy [3]. Many elderly patients will not be candidates for true TMT as they will not be cisplatin "fit". To date, no randomized controlled trials exist comparing RC to TMT and its unlikely that accrual numbers of such a trial would even be large enough to provide meaningful data. However, based on the best available data, TMT is a reasonable alternative to RC for select patients with the intention to perform a RC if there is no evidence of initial tumor response to TMT [3]. Patients considering TMT who desire to maintain a functional bladder, must accept that if they fail TMT, they will be far less likely to get either a orthotopic neobladder or continent diversion at time of salvage RC [86].

Patients with MIBC who are poor surgical candidates present a difficult management situation as their usually severe co-morbid conditions often prevent the usage of cisplatin required for effective TMT bladder sparing protocol. Several experimental protocols involving alternatives to cisplatin in combination with TURBT and radiation are underway [84]. Radical TURBT alone, or in conjunction with radiation, for MIBC patients unfit for surgery and cisplatin may have some success for those with a low tumor burden, no CIS, and no hydronephrosis. Patients with confirmed pT0 status on repeat TURBT within the first 3 months will likely have the most success.

Regardless, when comparing TURBT to RC in elderly patients, TURBT alone had a much worse cancer specific and overall survival [87]. Other retrospective series evaluating bladder preservation strategies have also shown worse cancer specific outcomes for elderly patients with MIBC compared to RC series $[88,89]$. And even though a strategy of repeat radical TURBT is less morbid than RC, it still has some limitations in the elderly. It requires strict adherence to cystoscopic evaluation every 3 months, at least initially, which can be a burden to both patient and family. Furthermore, patients may also require multiple procedures with anesthesia which is associated with cognitive dysfunction in the elderly [90].

Although TURBT is a routine procedure with low morbidity, the elderly with MIBC are at higher risk for bladder perforation. This is particularly true for females with a low BMI, usually because of thin bladder walls [91]. Those with a large tumor burden, which is most muscle invasive tumors, are also at increased risk of perforation [91, 92]. Fortunately, most perforations are extraperitoneal and can be managed with extended Foley catheter drainage, however intraperitoneal perforations can occur which requires emergent open exploratory surgery and repair. Elderly patients are at the greatest risk for perforations that require open surgical repair [93].

\section{Conclusion}

Elderly patients with muscle invasive bladder cancer are a unique and growing population. Several challenges must be overcome to improve care. The most important of which is finding an effective alternative to cisplatin that is better tolerated and can be given to those with renal insufficiency. Radical cystectomy remains the standard of care and is safe in older patients. Attempts should be made to optimize perioperative management to reduce the morbidity of the procedure. Immediate referral of elderly patients with MIBC to high-volume RC centers will help improve outcomes. Bladder sparing protocols using combination radical TURBT and chemo-radiation are currently limited by the lack of a good alternative to cisplatin, however bladder sparing does have promise for carefully selected patients.

Conflict of Interest Eugene J. Pietzak declares he has no conflict of interest.

George W. Drach declares he has no conflict of interest.

S. Bruce Malkowicz declares he has no conflict of interest.

Thomas J. Guzzo declares he has no conflict of interest.

\section{References}

Papers of particular interest, published recently, have been highlighted as:

- Of importance

-. Of major importance

1. Siegel R, Naishadham D, Jemal A. Cancer statistics, 2012. CA Cancer J Clin. 2012;62(1):10-29. Epub 2012/01/13.

2. Gundrum JD, Go RS. Cancer in the oldest old in the United States: current statistics and projections. J Geriatr Oncol. 2012;3:299-306.

3. Mak RH, Zietman AL, Heney NM, Kaufman DS, Shipley WU. Bladder preservation: optimizing radiotherapy and integrated treatment strategies. BJU Int. 2008;102(9 Pt B):1345-53. Epub 2008/11/28.

4. Herr HW, Dotan Z, Donat SM, Bajorin DF. Defining optimal therapy for muscle invasive bladder cancer. J Urol. 2007;177(2):437-43. Epub 2007/01/16.

5. Margel D, Alkhateeb SS, Finelli A, Fleshner N. Diminished efficacy of Bacille Calmette-Guerin among elderly patients with nonmuscle invasive bladder cancer. Urology. 2011;78(4):848-54. Epub 2011/08/16.

6. Gschwend JE, Retz M, Kuebler H, Autenrieth M. Indications and oncologic outcomes of radical cystectomy for urothelial bladder cancer. Eur Urol Suppl. 2010;9:10-8.

7. • Donat SM, Siegrist T, Cronin A, Savage C, Milowsky MI, Herr HW. Radical cystectomy in octogenarians-does morbidity outweigh the potential survival benefits? J Urol. 2010;183(6):21717. Epub 2010/04/20. The MSKCC experience with RC in elderly patients showing that $R C$ can be safe and effective at high volume centers.

8. Mendiola FP, Zorn KC, Gofrit ON, et al. Cystectomy in the ninth decade: operative results and long-term survival outcomes. Can J Urol. 2007;14(4):3628-34. Epub 2007/09/06. 
9. Tyritzis SI, Anastasiou I, Stravodimos KG, et al. Radical cystectomy over the age of 75 is safe and increases survival. BMC Geriatr. 2012;12:18. Epub 2012/05/02.

10. Farnham SB, Cookson MS, Alberts G, Smith Jr JA, Chang SS. Benefit of radical cystectomy in the elderly patient with significant co-morbidities. Urol Oncol. 2004;22(3):178-81. Epub 2004/07/24.

11. Pietzak E, Drach GW, Malkowicz SB, Guzzo T. Similar oncologic outcomes, but worse overall survival, for octogenarians following radical cystectomy. Abstract presented at 2012 Annual Meeting of Mid-Alantic Section of the American Urological Association. 2012.

12. Rink M, Dahlem R, Kluth L, et al. Older patients suffer from adverse histopathological features after radical cystectomy. Int $\mathrm{J}$ Urol. 2011;18(8):576-84. Epub 2011/06/28.

13. Nielsen ME, Shariat SF, Karakiewicz PI, et al. Advanced age is associated with poorer bladder cancer-specific survival in patients treated with radical cystectomy. Eur Urol. 2007;51(3):699-706. discussion -8. Epub 2006/11/23.

14. - Gray PJ, Fedewa SA, Shipley WU, et al. Use of potentially curative therapies for muscle-invasive bladder cancer in the United States: results from the national cancer data base. Eur Urol. 2012. Epub 2012/12/04. This study well demostrates the diasparity of care that most elderly patients experience.

15. Hollenbeck BK, Miller DC, Taub D, et al. Aggressive treatment for bladder cancer is associated with improved overall survival among patients 80 years old or older. Urology. 2004;64(2):292-7. Epub 2004/08/11.

16. Konety BR, Joslyn SA. Factors influencing aggressive therapy for bladder cancer: an analysis of data from the SEER program. J Urol. 2003;170(5):1765-71. Epub 2003/10/09.

17. Herr HW, Faulkner JR, Grossman HB, et al. Surgical factors influence bladder cancer outcomes: a cooperative group report. J Clin Oncol. 2004;22(14):2781-9. Epub 2004/06/17.

18. Horovitz D, Turker P, Bostrom PJ, et al. Does patient age affect survival after radical cystectomy? BJU Int. 2012. Epub 2012/05/04

19. Makary MA, Segev DL, Pronovost PJ, et al. Frailty as a predictor of surgical outcomes in older patients. J Am Coll Surg. 2010;210(6):901-8. Epub 2010/06/01.

20. • Lawrentschuk N, Colombo R, Hakenberg OW, et al. Prevention and management of complications following radical cystectomy for bladder cancer. Eur Urol. 2010;57(6):983-1001. Epub 2010/ 03/17. This review describes the morbidity associated with $R C$ and highlights areas for improvement.

21. Svatek RS, Fisher MB, Matin SF, et al. Risk factor analysis in a contemporary cystectomy cohort using standardized reporting methodology and adverse event criteria. J Urol. 2010;183(3):92934. Epub 2010/01/20

22. Stimson CJ, Chang SS, Barocas DA, et al. Early and late perioperative outcomes following radical cystectomy: 90-day readmissions, morbidity and mortality in a contemporary series. $\mathrm{J}$ Urol. 2010;184(4):1296-300. Epub 2010/08/21.

23. Taub DA, Dunn RL, Miller DC, Wei JT, Hollenbeck BK. Discharge practice patterns following cystectomy for bladder cancer: evidence for the shifting of the burden of care. J Urol. 2006;176(6 Pt 1):2612-7. discussion 7-8. Epub 2006/11/07.

24. Taylor JM, Feifer A, Savage CJ, et al. Evaluating the utility of a preoperative nomogram for predicting 90-day mortality following radical cystectomy for bladder cancer. BJU Int. 2012;109(6):8559. Epub 2011/07/05.

25. Abdollah F, Sun M, Schmitges J, et al. Development and validation of a reference table for prediction of postoperative mortality rate in patients treated with radical cystectomy: a population-based study. Ann Surg Oncol. 2012;19(1):309-17. Epub 2011/06/28.

26. - Morgan TM, Keegan KA, Barocas DA, et al. Predicting the probability of 90-day survival of elderly patients with bladder cancer treated with radical cystectomy. J Urol. 2011;186(3):82934. Epub 2011/07/27. An excellent retrospective analysis from a high volume center on predictiors of outcomes following $R C$ in a high risk group.

27. Mayr R, May M, Martini T, et al. Comorbidity and performance indices as predictors of cancer-independent mortality but not of cancer-specific mortality after radical cystectomy for urothelial carcinoma of the bladder. Eur Urol. 2012;62(4):662-70. Epub 2012/04/27

28. Mayr R, May M, Martini T, et al. Predictive capacity of four comorbidity indices estimating perioperative mortality after radical cystectomy for urothelial carcinoma of the bladder. BJU Int. 2012;110(6 Pt B):E222-7. Epub 2012/02/09.

29. van Leeuwen BL, Kristjansson SR, Audisio RA. Should specialized oncogeriatric surgeons operate older unfit cancer patients? Eur J Surg Oncol. 2010;36 Suppl 1:S18-22. Epub 2010/07/02.

30. Audisio RA, Pope D, Ramesh HS, et al. Shall we operate? Preoperative assessment in elderly cancer patients (PACE) can help. A SIOG surgical task force prospective study. Crit Rev Oncol Hematol. 2008;65(2):156-63. Epub 2007/12/18.

31. Pasetto LM, Lise M, Monfardini S. Preoperative assessment of elderly cancer patients. Crit Rev Oncol Hematol. 2007;64(1):10-8. Epub 2007/09/11.

32. Snyder C, Harlan L, Knopf K, Potosky A, Kaplan R. Patterns of care for the treatment of bladder cancer. J Urol. 2003;169(5):1697701. Epub 2003/04/11.

33. Prout Jr GR, Wesley MN, Yancik R, Ries LA, Havlik RJ, Edwards BK. Age and comorbidity impact surgical therapy in older bladder carcinoma patients: a population-based study. Cancer. 2005;104(8):1638-47. Epub 2005/09/01.

34. Herr H, Lee C, Chang S, Lerner S. Standardization of radical cystectomy and pelvic lymph node dissection for bladder cancer: a collaborative group report. J Urol. 2004;171(5):1823-8. discussion 7-8. Epub 2004/04/13.

35. Wilson CT, Woloshin S, Schwartz LM. Choosing where to have major surgery: who makes the decision? Arch Surg. 2007;142(3):242-6. Epub 2007/03/21.

36. Hamel MB, Lynn J, Teno JM, et al. Age-related differences in care preferences, treatment decisions, and clinical outcomes of seriously ill hospitalized adults: lessons from SUPPORT. J Am Geriatr Soc. 2000;48(5 Suppl):S176-82. Epub 2000/05/16.

37. Fahmy NM, Mahmud S, Aprikian AG. Delay in the surgical treatment of bladder cancer and survival: systematic review of the literature. Eur Urol. 2006;50(6):1176-82. Epub 2006/07/19.

38. Barbieri CE, Lee B, Cookson MS, et al. Association of procedure volume with radical cystectomy outcomes in a nationwide database. $\mathrm{J}$ Urol. 2007;178(4 Pt 1):1418-21. discussion 21-2. Epub 2007/08/21.

39. Gore JL, Litwin MS, Lai J, et al. Use of radical cystectomy for patients with invasive bladder cancer. J Natl Cancer Inst. 2010;102(11):802-11. Epub 2010/04/20.

40. Vora AA, Harbin A, Rayson R, et al. Alvimopan provides rapid gastrointestinal recovery without nasogastric tube decompression after radical cystectomy and urinary diversion. Can J Urol. 2012;19(3):6293-8. Epub 2012/06/19.

41. Schuster TG, Montie JE. Postoperative ileus after abdominal surgery. Urology. 2002;59(4):465-71. Epub 2002/04/03.

42. Pietzak E, Hwang W, Malkowicz SB, Guzzo T. Predictors of a prolonged length of hospital stay following radical cystectomy. Manuscript in Preparation. 2013.

43. Large MC, Reichard C, Williams JT, et al. Incidence, risk factors, and complications of postoperative delirium in elderly patients undergoing radical cystectomy. Urology. 2013;81(1):123-9. Epub 2012/11/17

44. Franco K, Litaker D, Locala J, Bronson D. The cost of delirium in the surgical patient. Psychosomatics. 2001;42(1):68-73. Epub $2001 / 02 / 13$ 
45. Marcantonio ER, Flacker JM, Wright RJ, Resnick NM. Reducing delirium after hip fracture: a randomized trial. J Am Geriatr Soc. 2001;49(5):516-22. Epub 2001/06/15.

46. Jacobs BL, Zhang Y, Tan HJ, Ye Z, Skolarus TA, Hollenbeck BK. Hospitalization trends after prostate and bladder surgery: implications of potential payment reforms. J Urol. 2013;189(1):59-65. Epub 2012/11/21.

47. Legner VJ, Massarweh NN, Symons RG, McCormick WC, Flum DR. The significance of discharge to skilled care after abdominopelvic surgery in older adults. Ann Surg. 2009;249(2):250-5. Epub 2009/02/13.

48. Aghazadeh MA, Barocas DA, Salem S, et al. Determining factors for hospital discharge status after radical cystectomy in a large contemporary cohort. J Urol. 2011;185(1):85-9. Epub 2010/11/16.

49. Goodney PP, Stukel TA, Lucas FL, Finlayson EV, Birkmeyer JD. Hospital volume, length of stay, and readmission rates in high-risk surgery. Ann Surg. 2003;238(2):161-7. Epub 2003/08/02.

50. Nelles JL, Joseph SA, Konety BR. The impact of marriage on bladder cancer mortality. Urol Oncol. 2009;27(3):263-7. Epub 2008/07/16.

51. Lawrence VA, Hazuda HP, Cornell JE, et al. Functional independence after major abdominal surgery in the elderly. J Am Coll Surg. 2004;199(5):762-72. Epub 2004/10/27.

52. Mathur S, Plank LD, Hill AG, Rice MA, Hill GL. Changes in body composition, muscle function and energy expenditure after radical cystectomy. BJU Int. 2008;101(8):973-7. discussion 7. Epub 2007/11/17.

53. Karl A, Staehler M, Bauer R, et al. Malnutrition and clinical outcome in urological patients. Eur J Med Res. 2011;16(10):469-72. Epub 2011/10/26.

54. Gregg JR, Cookson MS, Phillips S, et al. Effect of preoperative nutritional deficiency on mortality after radical cystectomy for bladder cancer. J Urol. 2011;185(1):90-6. Epub 2010/11/16.

55. Gibbs J, Cull W, Henderson W, Daley J, Hur K, Khuri SF. Preoperative serum albumin level as a predictor of operative mortality and morbidity: results from the National VA Surgical Risk Study. Arch Surg. 1999;134(1):36-42. Epub 1999/02/02.

56. Braga M, Gianotti L, Nespoli L, Radaelli G, Di Carlo V. Nutritional approach in malnourished surgical patients: a prospective randomized study. Arch Surg. 2002;137(2):174-80. Epub 2002/03/05.

57. Rodriguez AR, Lockhart A, King J, et al. Cutaneous ureterostomy technique for adults and effects of ureteral stenting: an alternative to the ileal conduit. J Urol. 2011;186(5):1939-43. Epub 2011/09/29.

58. Drewa T, Chlosta P, Czajkowski R. Will tissue-engineered urinary bladders change indications for a laparoscopic cystectomy? Surg Innov. 2010;17(4):295-9. Epub 2010/07/27.

59. Liao W, Yang S, Song C, et al. Tissue-engineered tubular graft for urinary diversion after radical cystectomy in rabbits. J Surg Res. 2012. Epub 2012/11/13.

60. Geutjes P, Roelofs L, Hoogenkamp H, et al. Tissue engineered tubular construct for urinary diversion in a preclinical porcine model. J Urol. 2012;188(2):653-60. Epub 2012/06/19.

61. Lau CS, Talug J, Williams SB, et al. Robotic-assisted laparoscopic radical cystectomy in the octogenarian. Int $\mathrm{J}$ Med Robot. 2012;8(2):247-52. Epub 2012/01/10.

62. Richards KA, Kader AK, Otto R, Pettus JA, Smith 3rd JJ, Hemal AK. Is robot-assisted radical cystectomy justified in the elderly? A comparison of robotic versus open radical cystectomy for bladder cancer in elderly $>/=75$ years old. J Endourol. 2012;26(10):13016. Epub 2012/05/16.

63. Challacombe BJ, Bochner BH, Dasgupta P, et al. The role of laparoscopic and robotic cystectomy in the management of muscle-invasive bladder cancer with special emphasis on cancer control and complications. Eur Urol. 2011;60(4):767-75. Epub 2011/05/31
64. Gore JL, Litwin MS. Quality of care in bladder cancer: trends in urinary diversion following radical cystectomy. World J Urol. 2009;27(1):45-50. Epub 2008/11/21.

65. Porter MP, Penson DF. Health related quality of life after radical cystectomy and urinary diversion for bladder cancer: a systematic review and critical analysis of the literature. J Urol. 2005;173(4):1318-22. Epub 2005/03/11.

66. Sogni F, Brausi M, Frea B, et al. Morbidity and quality of life in elderly patients receiving ileal conduit or orthotopic neobladder after radical cystectomy for invasive bladder cancer. Urology. 2008;71(5):919-23. Epub 2008/03/22.

67. Van der Aa F, Joniau S, Van Den Branden M, Van Poppel H. Metabolic changes after urinary diversion. Adv Urol. 2011;2011:764325. Epub 2011/06/21.

68. Bakke A, Jensen KM, Jonsson $\mathrm{O}$, et al. The rationale behind recommendations for follow-up after urinary diversion: an evidence-based approach. Scand J Urol Nephrol. 2007;41(4):2619. Epub 2007/09/01.

69. Raisz LG. Clinical practice. Screening for osteoporosis. N Engl J Med. 2005;353(2):164-71. Epub 2005/07/15.

70. Daneshmand S, Bartsch G. Improving selection of appropriate urinary diversion following radical cystectomy for bladder cancer. Expert Rev Anticancer Ther. 2011;11(6):941-8. Epub 2011/06/29.

71. Colwell JC, Gray M. Does preoperative teaching and stoma site marking affect surgical outcomes in patients undergoing ostomy surgery? J Wound Ostomy Continence Nurs. 2007;34(5):492-6. Epub 2007/09/19.

72. Tal R, Cohen MM, Yossepowitch O, et al. An ileal conduit-who takes care of the stoma? J Urol. 2012. Epub 2012/03/20.

73. - Houede N, Pourquier P, Beuzeboc P. Review of current neoadjuvant and adjuvant chemotherapy in Muscle-Invasive Bladder Cancer. Eur Urol Suppl. 2011;10:e20-5. Excellent overview of the history and current usage of perioperative chemotherapy.

74. Neoadjuvant chemotherapy in invasive bladder cancer: update of a systematic review and meta-analysis of individual patient data advanced bladder cancer (ABC) meta-analysis collaboration. Eur Urol. 2005;48(2):202-5; discussion 5-6. Epub 2005/06/09.

75. Tilki D, Svatek RS, Novara G, et al. Stage pT0 at radical cystectomy confers improved survival: an international study of 4,430 patients. J Urol. 2010;184(3):888-94. Epub 2010/07/21.

76. Sylvester R, Sternberg C. The role of adjuvant combination chemotherapy after cystectomy in locally advanced bladder cancer: what we do not know and why. Ann Oncol. 2000;11(7):851-6. Epub 2000/09/21.

77. Ruggeri EM, Giannarelli D, Bria E, et al. Adjuvant chemotherapy in muscle-invasive bladder carcinoma: a pooled analysis from phase III studies. Cancer. 2006;106(4):783-8. Epub 2006/01/19.

78. Skinner DG, Daniels JR, Russell CA, et al. The role of adjuvant chemotherapy following cystectomy for invasive bladder cancer: a prospective comparative trial. J Urol. 1991;145(3):459-64. discussion 64-7. Epub 1991/03/01.

79. Dash A, Galsky MD, Vickers AJ, et al. Impact of renal impairment on eligibility for adjuvant cisplatin-based chemotherapy in patients with urothelial carcinoma of the bladder. Cancer. 2006;107(3):506-13. Epub 2006/06/15.

80. Millikan R, Dinney C, Swanson D, et al. Integrated therapy for locally advanced bladder cancer: final report of a randomized trial of cystectomy plus adjuvant M-VAC versus cystectomy with both preoperative and postoperative M-VAC. J Clin Oncol. 2001;19(20):4005-13. Epub 2001/10/16.

81. David KA, Milowsky MI, Ritchey J, Carroll PR, Nanus DM. Low incidence of perioperative chemotherapy for stage III bladder cancer 1998 to 2003: a report from the National Cancer Data Base. J Urol. 2007;178(2):451-4. Epub 2007/06/15. 
82. • Galsky MD, Hahn NM, Rosenberg J, et al. A consensus definition of patients with metastatic urothelial carcinoma who are unfit for cisplatin-based chemotherapy. Lancet Oncol. 2011;12(3):211-4. Epub 2011/03/08. This consensus statement will help define the patient population to be targeted for enrollment in randomized studies to investigate alternatives to cisplatin.

83. Hutchins LF, Unger JM, Crowley JJ, Coltman Jr CA, Albain KS. Underrepresentation of patients 65 years of age or older in cancer-treatment trials. N Engl J Med. 1999;341(27):2061-7. Epub 1999/12/30.

84. Vaughn DJ. Chemotherapeutic options for cisplatin-ineligible patients with advanced carcinoma of the urothelium. Cancer Treat Rev. 2008;34(4):328-38. Epub 2008/02/12.

85. Bellmunt J, Petrylak DP. New therapeutic challenges in advanced bladder cancer. Semin Oncol. 2012;39(5):598-607. Epub 2012/10/09.

86. Eswara JR, Efstathiou JA, Heney NM, et al. Complications and long-term results of salvage cystectomy after failed bladder sparing therapy for muscle invasive bladder cancer. J Urol. 2012;187(2):463-8. Epub 2011/12/20.

87. Faba OR, Palou J, Urdaneta G, Gausa L, Villavicencio H. Invasive bladder cancer in the eighties: transurethral resection or cystectomy? Int Braz J Urol. 2011;37(1):49-55. discussion -6. Epub 2011/03/10.

88. Tran E, Souhami L, Tanguay S, Rajan R. Bladder conservation treatment in the elderly population: results and prognostic factors of muscle-invasive bladder cancer. Am J Clin Oncol. 2009;32(4):333-7. Epub 2009/04/22.

89. Wehrberger C, Berger I, Marszalek M, et al. Bladder preservation in octogenarians with invasive bladder cancer. Urology. 2010;75(2):370-5. Epub 2009/12/19.

90. Silverstein JH, Deiner SG. Perioperative delirium and its relationship to dementia. Prog Neuropsychopharmacol Biol Psychiatry. 2012;43C:108-15. Epub 2012/12/12.

91. Herkommer K, Hofer C, Gschwend JE, Kron M, Treiber U. Gender and body mass index as risk factors for bladder perforation during primary transurethral resection of bladder tumors. J Urol. 2012;187(5):1566-70. Epub 2012/03/20.

92. Collado A, Chechile GE, Salvador J, Vicente J. Early complications of endoscopic treatment for superficial bladder tumors. J Urol. 2000;164(5):1529-32. Epub 2000/10/12.

93. Golan S, Baniel J, Lask D, Livne PM, Yossepowitch O. Transurethral resection of bladder tumour complicated by perforation requiring open surgical repair - clinical characteristics and oncological outcomes. BJU Int. 2011;107(7):1065-8. Epub 2010/09/24. 